2015

\title{
Evaluation of Parallel Reduction Strategies for Fusion of Sensory Information from a Robot Team.
}

Damian M. Lyons

Fordham University, dlyons@fordham.edu

Joseph Leroy

Fordham University

Follow this and additional works at: https://fordham.bepress.com/frcv_facultypubs

Part of the Robotics Commons

\section{Recommended Citation}

Lyons, Damian M. and Leroy, Joseph, "Evaluation of Parallel Reduction Strategies for Fusion of Sensory Information from a Robot Team." (2015). Faculty Publications. 48.

https://fordham.bepress.com/frcv_facultypubs/48 


\title{
Evaluation of Parallel Reduction Strategies for Fusion of Sensory Information from a Robot Team.
}

\author{
Damian M. Lyons and Joseph Leroy \\ Fordham University, Robotics and Computer Vision Laboratory, Bronx NY 10458
}

\begin{abstract}
The advantage of using a team of robots to search or to map an area is that by navigating the robots to different parts of the area, searching or mapping can be completed more quickly. A crucial aspect of the problem is the combination, or fusion, of data from team members to generate an integrated model of the search/mapping area. In prior work we looked at the issue of removing mutual robots views from an integrated point cloud model built from laser and stereo sensors, leading to a cleaner and more accurate model. This paper addresses a further challenge: Even with mutual views removed, the stereo data from a team of robots can quickly swamp a WiFi connection.

This paper proposes and evaluates a communication and fusion approach based on the parallel reduction operation, where data is combined in a series of steps of increasing subsets of the team. Eight different strategies for selecting the subsets are evaluated for bandwidth requirements using three robot missions, each carried out with teams of four Pioneer 3-AT robots. Our results indicate that selecting groups to combine based on similar pose but distant location yields the best results.
\end{abstract}

Keywords: cognitive robotics, navigation, sensory fusion.

\section{INTRODUCTION}

Using a team of robots to quickly explore a disaster site offers a way to quickly assess damage, and bring timely care to injured victims with a lesser risk to first responders. In prior work, we have investigated how to quickly deploy a team of robots across a building [1] [2] as well as how to combine the sensory data from a team of robots working together so as to eliminate transient mutual views [3] [4]. The architecture used in these experiments is shown in Figure 1: As members of the robot team are dispersed through the building they all transmit their sensory data to a remote command center for registration, fusion and filtering. Consider a single robot streaming RGB-D sensory data to the command center. One frame of RGB-D data requires $(3 \times 4 \times 8)$ bits for location and $(1 \times 4 \times 8)$ bits for color per pixel. For a $640 \times 480$ image this is $39.3 \mathrm{Mb}$. A team of 10 robots transmitting at $20 \mathrm{fps}$ would require $7.8 \mathrm{Gbps}$. The current max bandwidth for $802.11 \mathrm{ac}$ is $1.7 \mathrm{Gbps}$ with a $7 \mathrm{Gbps}$ theoretical max. Clearly the approach shown in Figure 1 is not a practical one for large teams.

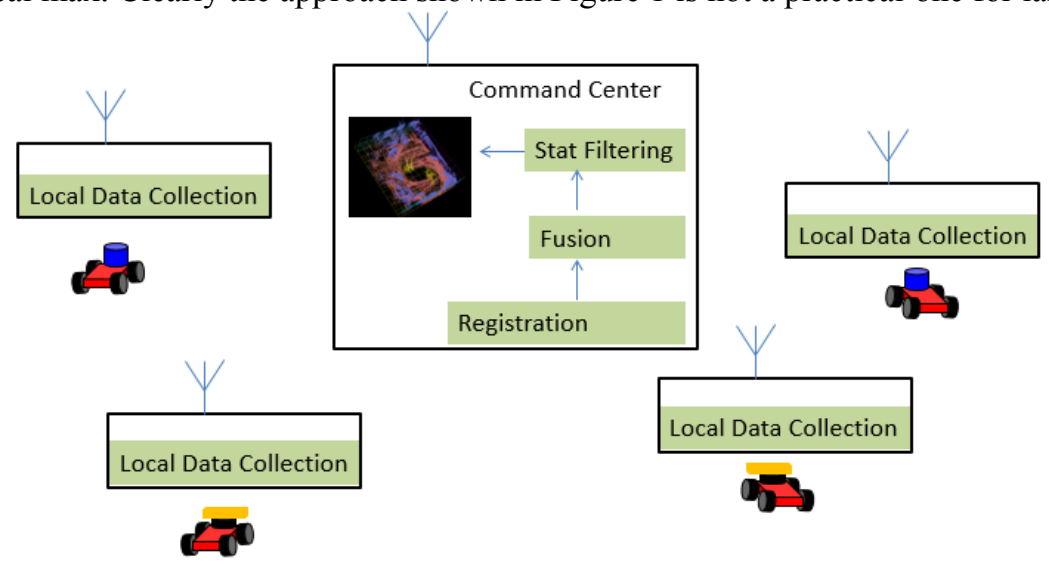

Figure 1: Robot Team Data Collection Architecture (from [4]).

The focus of multirobot exploration research has typically been on the exploration algorithm and on the mapping issues [5]. However, as Olson et al. [6] point out, communications and communication bandwidth become an issue for a 
large team of robots. For this reason, communication issues have also received some attention. Powers \& Balch [7] introduce a behavior-based, line-of-sight maintenance algorithm which they evaluated in simulation using MissionLab [8]. They present performance measurements such as the time to finish a mission, the percentage of time the team was fully connected and so forth. Burgard et al. [9] address the problem of loss of connectivity between the full team by simply applying their exploration algorithm to connected subgroups of the team, resulting, in the worst case, in each robot exploring all by itself. The issue of communication breakdowns also drives the work of Matignon et al. [10] who develop a Decentralized Markov Decision Process approach to maximizing exploration coverage under the communication constraints. They test their approach on a robot team equipped with laser scanners. Vissers and De Hoog [11] [12] propose a role-based approach to handling communication breakdown, where some robots perform as relays and some as explorers, relying on a rendez-vous schedule to coordinate with each other. They show in simulation that their approach explores space as quickly as an opportunistic (unconstrained by communication) approach, but returns data to the base station more quickly and more frequently. Many of these approaches rely on laser scanner data. However, as discussed, the use of RGB$\mathrm{D}$ sensors in the team raises a quite different communication problem to that previously studied: managing bandwidth as well as loss of communication.

This paper proposes a solution for this communication bottleneck problem by having pairs of robot team members communicate with each other using a proposed Team Communication by Reduction Algorithm (TCRA). The essence of the approach is that pairs of robot team-mates can communicate smaller messages to each other in parallel. When these messages are fused together, the resulting sensory data can potentially be smaller than the sum of each robot's message data because redundant data and noisy data can be eliminated by filtering and fusion. This fused data can then be combined with fused data from other pairs, filtered and fused again, and so on until a single combined dataset is ready to be passed to the remote command center. This process can occur faster than the broadcast solution of Figure 1 because pairs communicate, filter and fuse in parallel, and the resulting message may require less bandwidth because of the elimination of noise and redundancy.

There are multiple ways to select which pairs communicate, and this selection may influence the communication performance improvements that can be obtained. This paper presents an evaluation of eight different selection mechanisms based on selecting robots that are close or far from one another, or that are looking in different or similar directions. The broadcast implementation of Figure 1 was used to collect a set of time-stamped data files from a team of four robots traversing four different dispersion paths. Each selection method was applied in turn to this data, and the resulting messages sizes and reductions due to filtering and fusion calculated showing the theoretical performance improvement possible for each method.

The paper is laid out as follows. Section 2 presents TCRA and the pair selection algorithm in detail, discussing and motivating the eight different selection criteria proposed. Three different evaluation criteria are proposed: The total reduction in bandwidth per update for the entire team, the reduction in message size due to filtering and fusion, and the rate of area coverage. Section 3 presents the data collection architecture, the robots used in the team, and the three data sets on which performance was evaluated. Section 4 presents the results, and Section 5 presents the discussion and conclusions of the paper.

\section{SUBGROUP SELECTION AND PERFORMANCE METRICS}

A parallel programming reduction operation [13] is a logarithmic time technique for applying a binary associative operator to a list of values. The technique works by first applying the operator to pairs of values from the list in parallel, generating $\lceil n / 2\rceil$ results, and then repeating this step until there is only a single result left. This paper proposes taking the same approach to communications between members of the robot team (Figure 2).

Let us assume that at some point all $n$ robots in the team $R=\left\{r_{1}, \ldots, r_{n}\right\}$ are ready to transmit. Rather than all transmitting to the remote control center (as in Figure 1), each robot will now select a partner according to a selection criterion. Discussion of the details of the criterion is postponed for the present. Once a pair of robots is selected to communicate, they can decide which will send and which will receive based on the amounts of data they need to exchange.

Let $m\left(r_{i}\right)$ be the message size for robot $r_{i}$ in this first communication step. So $m\left(r_{i}\right)$ contains only the information collected by robot $r_{i}$ since the last communication event. If $P=\left\{r_{i}, r_{j}\right\} \subseteq R$, for $i \neq j$, is the selected pair of robots, then then transmitting robot $r_{t}$ is chosen as

$$
r t=\underset{r \in P}{\operatorname{argmin}} m(r) .
$$




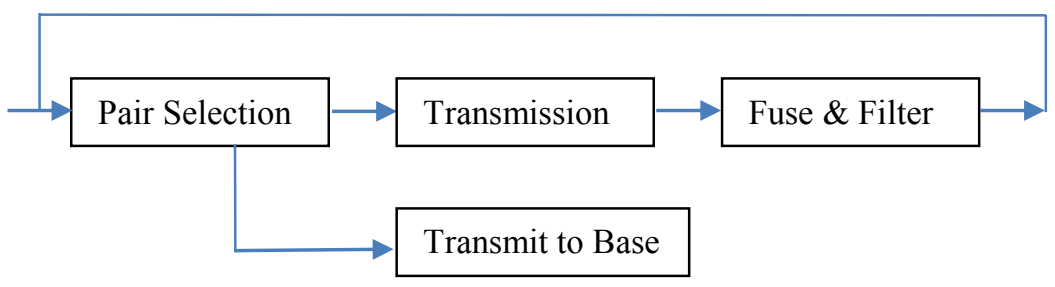

Figure 2: Proposed Team Communication by Reduction Algorithm (TCRA).

For a team of $n$ robots, this means that TCRA will take $\left\lceil\log _{2} n\right\rceil$ communication steps (communication levels) to assemble the full communication. Let $s \in\left\{1, \ldots,\left\lceil\log _{2} n 7\right\}\right.$ be the set of communication step numbers. At each step there will be a set of sets of communicating robots $P_{s}$ and the time for that step to finish will be dictated by the pair that took longest to communicate. Let $\mathrm{T}_{\mathrm{s}}$ be the set of the transmitted message sizes for all pairs in step $s$, defined as:

$$
T_{s}=\left\{m(r t) \mid r t=\underset{r \in P}{\operatorname{argmin}} m(r), P \in P_{s}\right\} .
$$

While reducing bandwidth consumption, the pair transmission method of eq. (1) is not very robust as it assumes there will only be one robot that has the final model, and which then needs to transmit it to the remote control center. As a first step however, we will restrict our attention in this paper to the single transmission per pair rather than a more robust exchange model, where pairs exchange their data messages.

Since all the communications at step $s$ happen in parallel, the time for the communication is related to the longest message, that is, to the maximum value of $m(r t)$ in $T_{s}$ for that step $s$, eq. (2). Each receiver carries out filtering on the data to remove overlap and noise, potentially improving the quality and reducing the size. The selection and communication process continues, with subgroups combining their information in parallel, until there is only one robot left. At this point that robot can transmit to the remote control center. This algorithm is show in Figure 2. The total communication time, therefore, is the sum of each such maximum for all steps (divided by the network bandwidth, but that scaling factor is omitted for simplicity):

$$
T=\sum_{s=1}^{s=\left[\log _{2} n\right\rceil} \max _{t \in T_{s}} t .
$$

Note that even without any reduction due to fusion or filtering, eq.(3) always yields a shorter communication time than the broadcast method, which would just yield $\mathrm{T}=\sum_{r \in R} m(r)$.

The same approach could be used for subgroups of size 3 or greater. While that reduces the number of communication steps, it introduces extra complexity into the subgroup selection algorithm and also increases the subgroup bandwidth requirements. We will only employ the pair selection approach here.

\subsection{Selection Criteria}

It is possible to suggest several different criteria for selecting the pairs that communicate. For example, the closest pair of robots could communicate first. Robots that are close may be viewing the same part of the scene, and hence fusion of their sensory data may improve the quality and reduce redundancy in the scene model. Furthermore, robots that are closer are less likely to have their communication attenuated or degraded. For example, in Figure 3, robot pair $\{r 1, r 2\}$ are the closest pair, and $\{r 3, r 4\}$ would be the second pair. However, note that $r 1$ and $r 2$ are looking in different directions; perhaps fusing $r 1$ and $r 3$, which are looking at the same part of the scene, might produce a smaller resultant model and thereby reduce the overall time of the communication event, eq. (3). More drastically, perhaps fusing distant robots that are viewing different parts of the scene might yield a better time in eq. (3) by more quickly assembling the full spatial range of the model (e.g., $r 2$ and $r 4)$. 


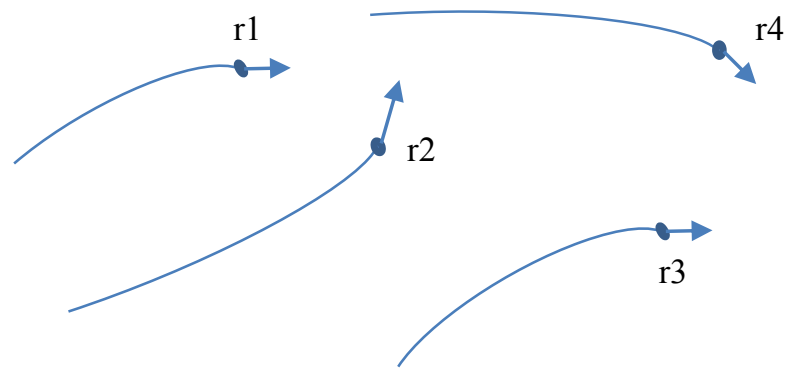

Figure 3: Selection Criteria Example. Tracks and current location and pose are shown for robot team $R=\{r 1, r 2, r 3, r 4\}$ during a team communication event. The location is denoted by the solid disk and orientation by the arrow at the end of each track

Based on this reasoning, a set of eight possible selection criteria are proposed here for evaluation. All the criteria are restricted for simplicity to selecting pairs of communicating robots. The following are the proposed selection criteria and their motivation:

1. Nearest team members communicate: Close team members are likely to be able to communicate without issues of interference or attenuation. They are also likely to be viewing the same part of the scene, and fusion of their information will reduce overlap and redundancy in data communications.

2. Nearest team members with similar pose communicate: Close team members may be looking at the same part of the scene or different parts of the scene. Restricting communication to those with similar pose should improve the overlap and hence reduce future communications.

3. Closest team members with dissimilar pose communicate: Combining team members with dissimilar views may not reduce overlap, but it may result in more quickly assembling a model that covers a wide spatial range.

4. Farthest team members communicate: Although more distant team members may experience issues of interference or attenuation, and may not have significant overlap, combining their data will produce a model with larger spatial range.

5. Farthest team members with similar pose communicate: Distant team members may be looking at the same part of the scene or different parts of the scene. Restricting communication to those with similar pose should improve the overlap while also improving spatial range.

6. Farthest team members with dissimilar pose communicate: Combining distant team members with dissimilar views should result in the quickest assembly of a model that covers a wide spatial range.

The implementation of each of these selection criteria for the purpose of the experimental evaluation in this paper will be described in Section 3. When two team members are selected to communicate, no matter the selection criterion, they carry out the following steps:

1. The team member with the smaller data message is considered the sender, and the one with the larger data message the receiver.

2. Each receiver combines the received data and its own data with a voxel filter, to eliminate any redundancy in the points collected, and secondly with a statistical filter, to reduce noise.

3. The team member with the smaller communication, the sender, no longer participates in the process, whereas the receiver goes on to the next level communication unless there are no more team members left.

4. If there are no more team members left, then the final receiver transmits the model to the remote control center.

\subsection{Performance Metrics}

A set of performance metrics are proposed to help evaluate the benefit of each of the selection criteria. During a robot mission, there will be numerous team communication events; Let $N_{c}$ be the number of communication events. The principal metric is the sum of the message sizes at each communication level (eq.(3)) versus the sum of message sizes for all team members, $\sum_{r \in R} m(r)$. This ratio is averaged over all $N_{c}$ for the mission. Additional metrics are also introduced to capture the data reduction due to spatial overlaps and the growth of the spatial range of the combined models. These metrics are again overaged over $N_{c}$ for the robot mission. 
To evaluate the performance of the various subgroup selection algorithms, the following set of performance criteria are proposed:

1. The average improvement in communication time $M$ across all communication events. The average sum of the messages sizes for all robots $M_{\text {all }}$ is the average of $\sum_{r \in R} m(r)$ over all communication events. The average sum of message sizes for each step in the subgroup selection and combination $M_{T C R A}$ is the average of $\mathrm{T}$ in eq. (3) over $N_{c}$ for the robot mission.

$$
M=\frac{M_{T C R A}}{M_{\text {all }}}
$$

2. The average data reduction $D$ across all communication events. For each pair of communicating robots $P=\left\{r_{i}\right.$, $\left.r_{j}\right\}$, the data reduction is the size of the filtered and fused combination divided by $m\left(r_{i}\right)+m\left(r_{j}\right)$. This value is averaged for each step of the communication event, yielding $\mathrm{D}_{\mathrm{s}}$. The final value for $\mathrm{D}$ is sum of the average at each step times the number of pairs at that step averaged over $N_{c}$ for the robot mission:

$$
D=\frac{1}{\sum_{s}\left|T_{s}\right|} \sum_{s=1}^{s=\left[\log _{2} n\right]}\left|T_{s}\right| D_{s}
$$

3. The average growth of spatial area $A$ across all communication events. The spatial area for each message is $a(r)$. For each communication pair, the overlap ratio $o v\left(r_{i}, r_{j}\right)$ is $a\left(r_{i}\right) \cap a\left(r_{j}\right)$ divided by the spatial area of the fused and filtered result. This value is averaged for each step of the communication event, yielding $A_{s}$. The final value for $A$ is the sum of the differences in overlap per level divided by the numbers of levels minus 1 and averaged over $N_{c}$ for the robot mission.

$$
A=\frac{1}{\left\lceil\log _{2} n\right\rceil-1} \sum_{s=1}^{s=\left[\log _{2} n\right\rceil-1}\left(A_{s+1}-A_{s}\right)
$$

It is expected that $M$ will show an improvement (i.e., $0<M<1.0$ ) in every case, since the parallel reduction strategy will produce a time improvement even without any reduction due to filtering and fusion. However, the different selection methods may produce different improvements. The data reduction $\mathrm{D}$ will also be in the range $0<D<1.0$ but this number will be larger for selection methods that have resulted in fusion and filtering reducing the combined scene model by the elimination of redundancy and noise. Finally the growth in overlap will be positive where there is smaller overlap at the early steps of a communication event, and negative where there is larger overlap in the early stages of the communication event. The will be smaller in absolute magnitude when there is little difference in the overlap at every level and larger when the range increases strongly between levels.

\section{DATA COLLECTION ARCHITECTURE}

The data collection architecture used to evaluate TCRA is the same as that used in prior work [4] [3] to evaluate mutual view elimination in robot teams operating in a confined area. We have constructed a straight-forward software architecture to investigate issues that arise in this kind of application, shown in Figure 1. Our robot teams consist of four robots, all Pioneer 3-AT models - two robots with tilted SICK LMS 200 lasers and two with Bumblebee 2 stereo-cameras mounted on a Directed-Perception pan-tilt base. The two laser-based robots have the lasers mounted in different configurations. Both laser-based robots and their laser configurations are shown in Figure 4. We have used a simple modification of the common horizontal laser configuration to allow us to generate 3-D information as a robot moves: a single, tilted laser. The complementary nature of these two laser robots and their use in constructing a 3D model has been described in [3]. All the robots have onboard computers and are programmed using the Aria toolkit in $\mathrm{C}++$. The client-server architecture of Figure 1 is implemented using the ArNetworking library of Aria to allow joystick control of multiple robots and asynchronous data collection from each robot. 

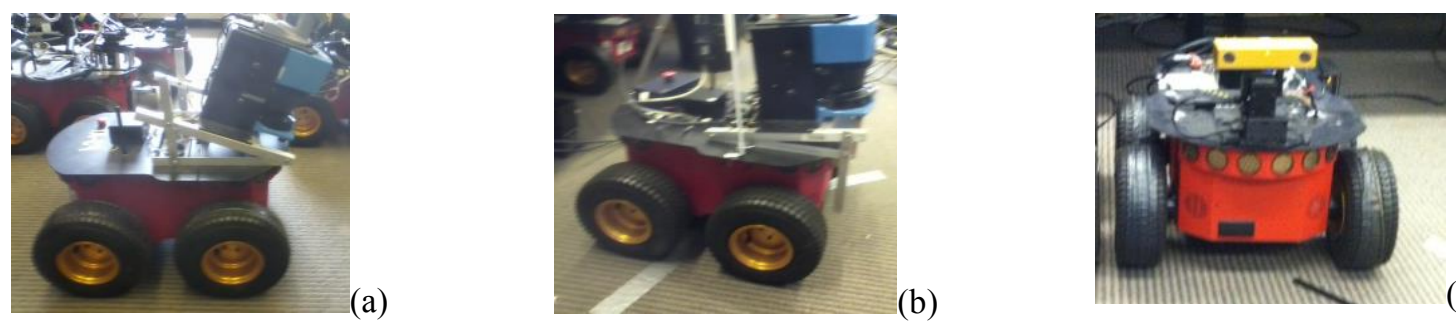

(c)

Figure 4: Robots used in data collection experiments.

(a) Down laser mounted on Pioneer 3-AT; (b) Up laser mounted on Pioneer 3-AT

(c) Stereo-camera mounted on Pioneer 3-AT

Three different robot missions were carried out, each consisting of a team of four robots exploring an indoor lab space of total size $6 m \times 10 m$. The robots were driven under manual control to a series of preplanned waypoints, while transmitting their sensory data to a central server at $1 \mathrm{~Hz}$ (laser robots) and $0.2 \mathrm{~Hz}$ (stereo robots). All the data was timestamped and stored separately along with the robot paths on the central server. The server time-stamps and stores all the data in the Point Cloud Library (PCL) format - for later analysis or inspection. It registers data from each robot based on the accompanying pose information, which is Kalman-filtered to account for uncertainty in robot motion.

The TCRA communication was emulated by traversing the dataset that had been stored for each mission off-line. The time stamp information was used to determine when the team was ready for a communication event, i.e., when all robots were ready to communicate. For each such event, the location and pose information was used to construct a distance matrix $D T$ and a pose difference matrix $P T$ as follows:

$$
\begin{array}{ll}
D T=\left[d t_{i j}\right] & \text { where } d t_{i j}=\text { distance from } r_{i} \text { to } r_{j} \\
P T=\left[p t_{i j}\right] & \text { where } p t_{i j}=\text { angular pose difference between } r_{i} \text { and } r_{j}
\end{array}
$$

The near (or far) distance criterion was evaluated by selecting the smallest (or largest, respectively) distances from $D T$. The similar (or dissimilar) pose criterion is evaluated in the same way from $P T$. The joint criteria (e.g., nearest with similar pose etc) was evaluated in a two-step process:

1. $P T$ is scanned to determine a set of similar (or dissimilar) pose robots, defined for the purpose of this experiment as a pose difference less than (or greater than, respectively) 20 degrees.

2. This list is evaluated to find the nearest (or farthest) robot pairs by looking for the smallest (or largest, respectively) value in $D T$.

The metrics in eqs. (4) - (6) were collected and averaged across every communication event in the mission $N_{c}$.

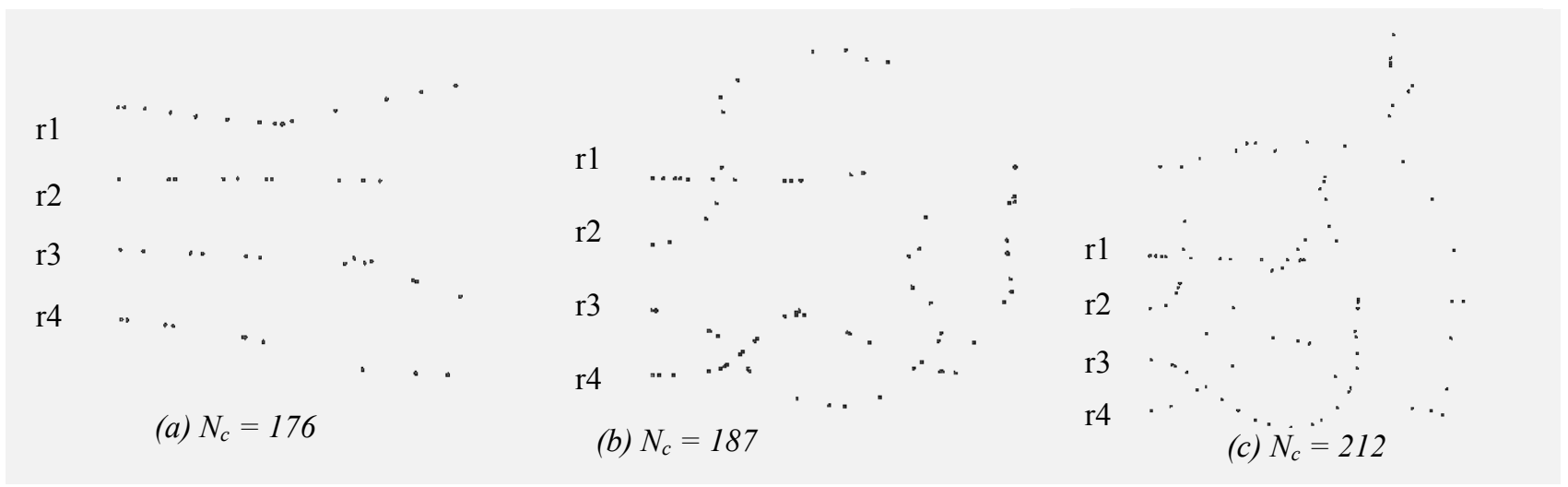

Figure 5: Robot paths for the 4 robot team for each of the three datasets (a) through (c) along with the number of communication events $\mathrm{N}_{\mathrm{c}}$ for each.

\section{RESULTS}

The robot paths for each of the three, indoor datasets are shown in Figure 5(a) through (c). Each point on the path for each robot $(r 1, r 2, r 3, r 4)$ is a time-stamped communication event. Robot $r 1$ and $r 4$ are laser robots (up and down-tilted laser, respectively) and robots $r 2$ and $r 3$ are stereo-camera robots. The first dataset, Figure 5(a), has 176 communication events and basically consists of all four robots moving forwards at different speeds. The second dataset, Figure 5(b), has 187 
communication events and consists of pairs of adjacent robots curving around each other at different rates. The final dataset, Figure 5(c), has 212 communication events and consists of all four robots winding around each other.

The robot mission shown in Figure 5(a) was the shortest. Figure 6(a) shows the final fused and filtered scene model for this mission, and Figure 6(b) shows the graph of the value of the communication performance improvement $M$ (eq.(4)) but for each communication event for all 8 selection criteria. While this graph shows a lot of information, it is clear that the communication performance increases as the mission proceeds. The mission begins with all strategies showing a communication performance improvement between 0.4 and 0.5 (i.e., message size is between $40 \%$ and $50 \%$ of the message size where all robots simply transmit their data, $M_{\text {all }}$ in eq. (4)). But as time proceeds and the robots drive into the scene and collect data, the communication performance improvements get even better as redundancy between the robots' measurements are leveraged - but equally not for all strategies. The final communication events show an improvement between 0.1 and 0.2 . Although there is an extremely strong improvement overall, some strategies have better performance than others.
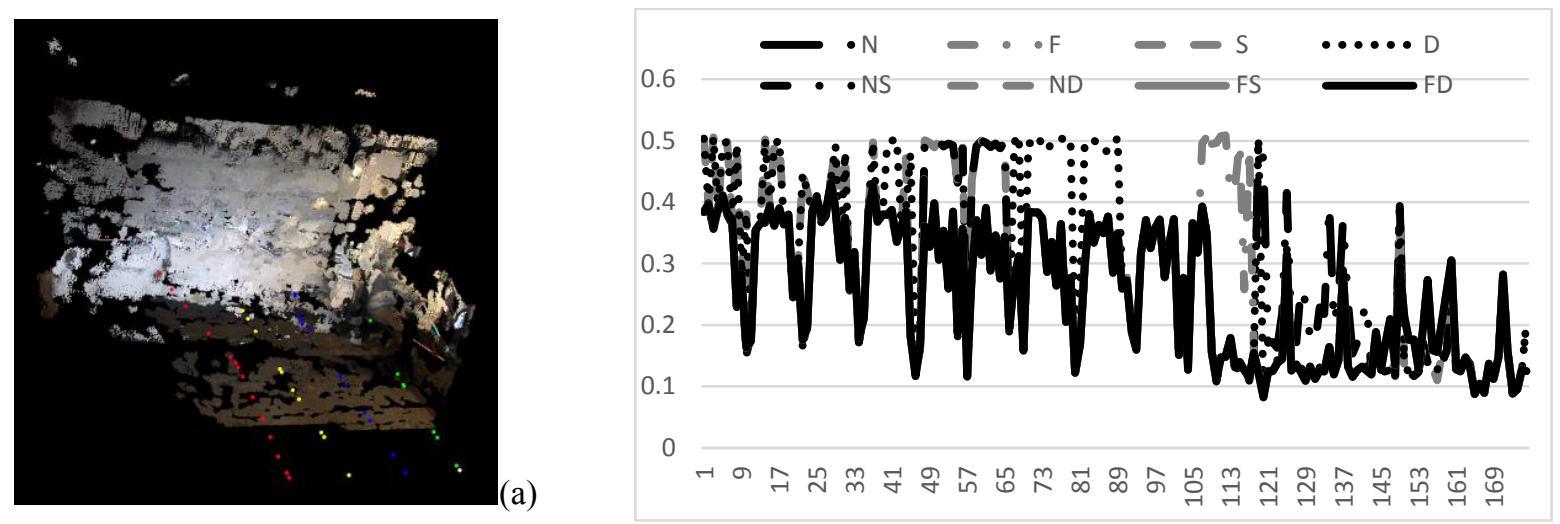

Figure 6: Some results from the first mission (Fig. 5(a)): The scene model is shown in (a) and the communication performance improvement per communication event for each of the eights election strategies is shown in (b).

For this reason, we calculated the average value of the three metrics from eqs. (4-6) and plotted them as a bar chart. The average values are shown in Figure 7. Figure 7(a) shows the average value for all 3 metrics for the first mission. So, the bar labelled ' $M$ ' on Figure 7(a) shows the average value of the communication performance improvement for the first mission, dataset 1, shown in detail in Figure 6(b). Of course the performance improvement might be a function of the mission itself - how the robots moved, and what geometry was measured - so this same average information was collected for the two other missions and show in Figure 7(b) (dataset 2) and figure 7(c) (dataset 3). Each bar chart in Figure 7 shows the three metrics, communication improvement $M$, data reduction $D$, and growth of spatial range $A$ for all eight strategies. The final chart, Figure 7(d) is the average of averages for all three missions.

The strategies with best communication performance are the similar pose (S) and farthest robots with similar pose (FS). The more obvious assumption would be that robots that are close would yield better communication performance. However, the conclusion for this admittedly small sample of indoor scenes is that similar pose is more important than similar position. And that the combination of most distant pairs with similar pose are better than closer pairs with similar performance.

Similarly for the data reduction metric, the best performance comes from combining near robots with dissimilar pose, or distant robots with similar pose. It could be argued that as with many types of fusion, combining dissimilar sources can yield a better result.

The area growth metrics are more difficult to interpret. A negative value means the spatial range grows later in the communication event, and a positive value means the range grew at the early stages of the communication. The magnitude indicates how much different there was in spatial range between the early and later stages of the communication event. For example, Figure 7(d) shows that the largest magnitude was for the FS strategy and that it was negative. This means that the full spatial range for the geometry was constructed very early in the communication - the strategy generates a very quick overview of the final result of the communication event. The smallest value of this metric was for the $\mathrm{N}$ strategy meaning the spatial range was pretty even for all events, so the spatial range for the geometry grew evenly across all communications. 


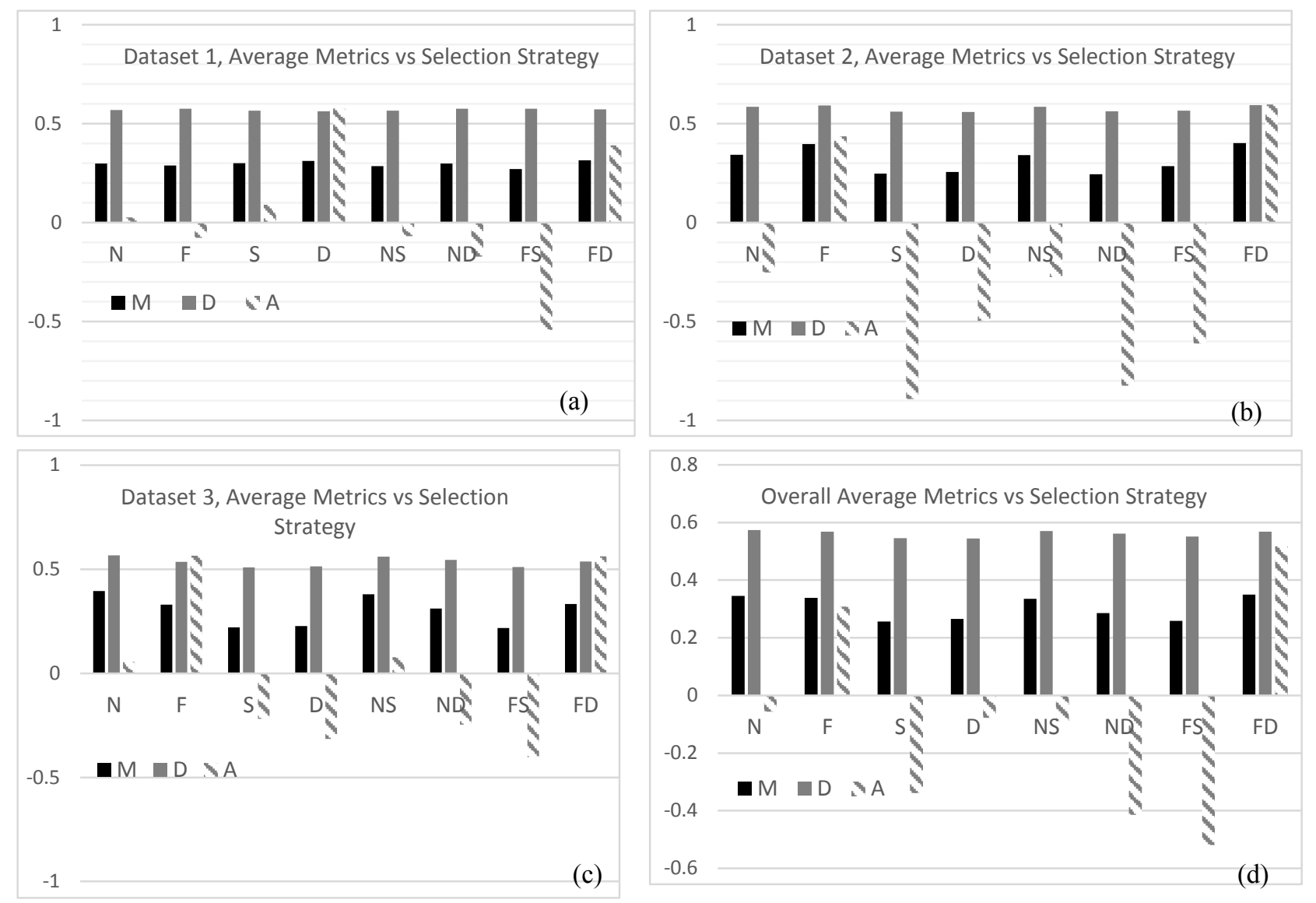

Figure 7: Averaged metrics for the three missions of Figure 5(a) through (c) shown in Figures 7(a) through (c) respectively as bar charts, with different color bars representing the communication improvement $M$, data reduction $D$, and growth of spatial range $A$ metrics, and the eight selection strategies as the horizontal axis. Figure 7(d) shows the overall averages for all missions combined.

\section{CONCLUSIONS}

When a large team of robots is deployed to a site with the objective of constructing a model of the site as fast as possible, within minutes of deployment if possible, and to make the model available for situational awareness at a remote command center, a number of issues arise. While the literature contains many examples of exploration algorithms and SLAM algorithms for this problem, the difficulties of communication among team members are not as widely addressed. Prior work has looked at the impact of communication constraints between robot team members on the exploration strategy and efficiency. For teams where the primary sensor is laser, the communication bandwidth is not particular stressed and a common solution is just to compress the data [6]. However, when RGB-D sensors are used, such as the Kinect or, as in our case, stereo-cameras, the volume of data transmitted by even a small team can overwhelm the available bandwidth, even for the latest 802.11 standards. This paper has propose and evaluated a solution to this communication bottleneck in team exploration.

The reduction algorithm, common in parallel processing [13], is the core of the Team Communication by Reduction Algorithm (TCRA) presented here. Pairs of robots communicate in parallel, combining their sensory data. The process is continued hierarchically until all the data has been combined in a single robot, requiring $\log _{2} n$ levels, where $n$ is the number of robots. Eight different strategies for selecting pairs were evaluated experimentally. The strategies were based on whether the pair had similar or dissimilar pose, and were near or far from each other. In prior work on the communication issues for teams of robots, the performance metric has typically been related to the exploration strategy: how much of the site is covered and how quickly. Our focus here however is primarily on the bandwidth required for communication events, so our metrics include by how much bandwidth requirements are reduced by each communication event, by how much bandwidth requirements are reduced when each pair combines information, and by how quickly the overall geometry is assembled during the communication. 
The strategies are evaluated on a set of three missions, each consisting of four robots, two with lasers and two with stereo-cameras, and carried out via teleoperation within a lab setting. The average values for the three metrics are presented for each mission and also combined into a single averaged chart. The results indicate that combining pairs by similarity in pose produces the best performance improvements. While it might arguably be intuitive to combine information from the closest pairs of robots, our results indicate that the best performance comes from combining the most distant robots with similar pose.

The results presented here are for three missions carried out by a team of four robots in an indoor setting. These results need to be validated for larger teams, for outdoor as well as indoor scenarios and for longer missions. Furthermore, although the experimentation was completed with physical robots, all the data was simply sent to a single central server and timestamped. The selection and reduction was then carried out on the server, simulating each of the strategies in turn and calculating the metrics. In this manner, we have identified pose similarity, and most distant robot, as the most useful selection criteria. The next step in this work is to implement this strategy using ad-hoc networking for each robot in the team.

\section{Bibliography}

[1] T.-L. Liu and D. Lyons, "Leveraging Area Bounds Information," in 13th Int. Conf. on Intelligent Autonomous Systems, Padua Italy, 2014.

[2] A. Kenealy, N. Primiano, A. Keyes and D. Lyons, "Thorough Exploration of Complex Environments with a Space-based Potential Field," in SPIE Intelligent Robotics and Computer Vision, San Francisco CA, 2016.

[3] D. Lyons, K. Shesthra and T.-L. Liu, "Fusion of Ranging Data from Robot Teams Operating in Confined Areas," in SPIE Defense, Security and Sensing: Multisource, Multisensor Information Fusion, Baltimore MD, 2013.

[4] D. Lyons and K. Shrestha, "Eliminating Mutual Views in Fusion of Ranging and RGB-D Data from Robot Teams Operating in Confined Areas," in SPIE Defense, Security and Sensing: Multisource, Multisensor Information Fusion, Baltimore MD, 2014.

[5] R. Reid and T. Bräunl, "Large-scale Multi-robot Mapping in MAGIC 2010," in IEEE Robotics, Automation and Mechatronics, Qingdao China, 2011.

[6] E. Olson et al, "Progress Towards Multirobot Reconnaissance and the MAGIC 2010 Competition," Journal of Field Robotics, vol. 29, no. 5, pp. 762-792, 2012.

[7] M. Powers and T. Balch, "Value-based Communication Preservation for Mobile Robotics," in 7th int. Symp. on Distributed Autonomous Robotic Systems (DARS), 2004.

[8] D. MacKenzie, R. Arkin and R. Cameron, "Multiagent Mission Specification and Execution," Autonomous Robots, vol. 4, no. 1, pp. 29-52, 1997.

[9] W. Burgard, M. Moors, C. Stachniss and F. Schneider, "Coordinated Multirobot Explroration," IEEE Trans on Rob., no. 21, pp. 376-386, 2005.

[10] L. Matignon, L. Jeanpierre and A. Mouaddib, "Coordinated multi-robot exploration under communication constraints using decentralized markov decision processes," in AAAI, 2012.

[11] A. Visser, J. de Hoog, A. Jimenez-Gonzalez and J.-R. Martinez de-Dios, "Discussion of Multirobot Exploreation in Communication Limited Environments," in Workshop on Towards Fully Decentralized Multi-Robot Systems: Hardware, Software and Integration, ICRA 2013, Karlsruhe Germany, 2013.

[12] J. de Hoog, S. Cameron and A. Visser, "Dynamic Team Hierarchies in Communication Limited Multirobot Exploreation," in International Workshop on Safety, Security and Rescue Robotics (SSRR), 2010.

[13] D. Lyons, Cluster Computing for Robotics and Computer Vision, Singapore: World Scientific, 2011. 